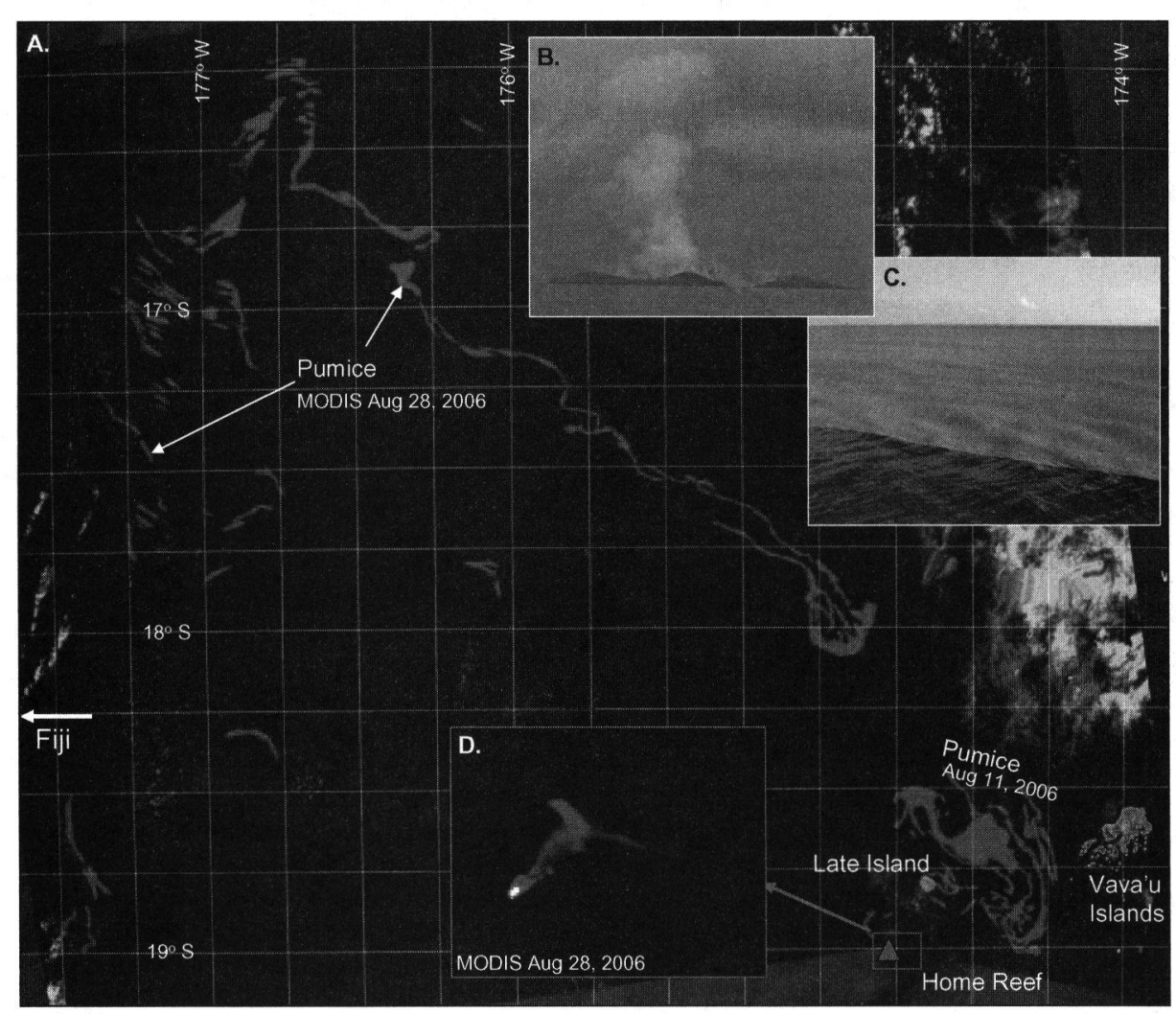

Fig. 2. (a) Extent of pumice rafts measured by the Moderate Resolution Imaging Spectroradiometer (MODIS) as of 11 August (lower right) and 28 August 2006 (upper left). Photographs of (b) new Home Reef island from about 3 kilometers away and of (c) floating pumice, taken by the yacht crew on 12 August 2006 (courtesy of FFransson). (d) Discolored water drifting away from Home Reef observed by MODIS on 28 August 2006. Original color image appears at the back of this volume.

Goddard DAAC. Thanks to the Smithsonian Institution Global Volcanism Program and the volcano listserv staff at Arizona State University for sharing information and supporting the open dialog between numerous scientists studying this eruption. Special thanks to Fredrik Fransson and the crew of the Maiken for their excellent observations new island. and photographs of the pumice rafts and

\section{References}

Bryan,S.E.,A.Cook, J.P.Evans, P.W.Colls, M. G.Wells, M. G Lawrence, J.S. Jell,A. Greig, and R. Leslie (2004), Pumice rafting and faunal dispersion during 2001-2002 in southwest Pacific: Record of a dacitic submarine explosive eruption from Tonga,Earth Planet. Sci. Lett., 227, 135-154.

Matangi Tonga Online (2006), New island emerges in Tonga, 6 Nov. (Available at http://www.matangitonga. to/article/tonganews/disasters/new_island061106. shtml)

Rothery,D.A., P.W.Francis, and C.A.Wood (1988),Volcano monitoring using short wavelength infrared data from satellites,J. Geophys. Res., 93(B7), 7993-8008.

Salomonson,V.,W. Barnes, P.Maymon, H. Montgomery and H.Ostrow (1989), MODIS: Advanced facility instrument for studies of the Earth as a system, IEEE Trans. Geosci. Remote Sens. 27(4), 145-153.

Smithsonian Institution (1984), Home Reef,Sci. Event Alert Network Bull., 9(2), Global Volcanism Program. (Available at http://www.volcano.si.edu/world/volcano cfm?vnum=0403-08=\&volpage=var\#sean 0904)

Smithsonian Institution (1985), Home Reef,Sci. Event Alert Network Bull., 10(3). (Available at http://www. volcano.si.edu/world/volcano.cfm?vnum=040308=\&volpage=var"tsean_0904)

Smithsonian Institution (2006), Home Reef, Global Volcanism Network Bull., 31(11). (Available at http:// www.volcano.si.edu/reports/usgs/index.cfm?content $=$ archive\&volcano=homereef)

Urai, M., and S. Machida (2005), Discolored seawater detection using ASTER reflectance products: A case study of Satsuma-Iwojima, Japan, Remote Sens. Environ., 99, 95-104.

Yamaguchi,Y.A. B. Kahle,H.Tsu,T.Kawakami, and M. Pniel (1998), Overview of Advanced Spaceborne Thermal Emission and Reflection Radiometer (ASTER), IEEE Trans. Geosci. Remote Sens., 36(4), 1062-1071.

\section{Author Information}

R. Greg Vaughan, Michael J.Abrams, Simon J. Hook and David C. Pieri, Jet Propulsion Laboratory, Califor nia Institute of Technology, Pasadena; E-mail: greg. vaughan@jpl.nasa.gov

\title{
Connecting the Sun to the Heliosphere
}

\section{PAGES 37,42}

To better understand the processes that connect the Earth environment to the Sun, NASA's Living With a Star program must address questions that cut across traditional science disciplines. To meet this challenge, the Targeted Research and Technology component of Living With a Star has developed an innovative strategy to focus research on the most complex science targets. The targets are drawn up each year by a panel of senior scientists. These targets are then publicized, and individual researchers can propose solutions. NASA then selects teams of researchers through a peer-review process for each targeted effort. This contrasts with other NASA programs in which individual and independent programs are selected for study.

T. H. ZURBUCHEN
This article reports on the science motivation and approach of one key team selected in September of 2005 to address the following major question: What determines the topology and evolution of the magnetic fields that stretch from the surface of the Sun to the outer boundary of the heliosphere?

\section{Heliospheric Magnetic Field}

The heliosphere is a teardrop-shaped bubble of hot gas, or plasma, in interstellar space that extends from the Sun to beyond 100 times the Sun-Earth distance (100 astronomical units (AU)). It is inflated by the solar wind and threaded by magnetic fields that affect the electrical environment of Earth and all the planets.

Events in the Earth's space environment are dominated by what happens at the inner heliospheric boundary, the solar corona. The spectacular observations of the Sun and its million-degree corona obtained by decades of ground-based observatories and by the Solar and Heliospheric Observatory (SOHO) and other spacecraft have attracted the attention of specialist and lay audiences alike. Yet, relatively little is known about the fundamental processes taking place within the corona.

One important reason that such issues have not been addressed is that it is not currently possible to measure the magnetic fields in the solar corona directly. In the mid-1970s, the two Helios spacecraft, developed by Germany and the United States to study the Sun, came within 0.29 $\mathrm{AU}$, or 62 solar radii $\left(R_{S}\right)$, of the Sun. This is the closest approach yet, but it is not close enough: The solar wind is heated and accelerated within $5 R_{S}$, and the supersonic solar wind decouples from the Sun at $10-20 R_{S}$ (the so-called Alfvén radius, where the speed of transverse waves in the magnetized plasma equals the solar wind speed itself). Helpful optical and radio wave observations of solar wind components in the corona exist, but they usually 
Eos, Vol. 88, No. 4, 23 January 2007

involve averaging the properties of many components in a long line of sight, and they are not capable of measuring the heliospheric magnetic field directly.

\section{Science Questions}

The challenges that face the Heliospheric Magnetic Field Targeted Research and Technology Focus Team can be summarized in four specific questions.

The first question is: What is the spatial distribution of open magnetic flux in the corona? Most magnetic fields in the corona are loop-like; they evidently close back on the solar surface at relatively low altitudes, as shown in Figure 1. Other fields appear to stretch into the heliosphere, but loops are easier to observe than the so-called open flux, because heated material is trapped in them and is not able to escape into the heliosphere. The 'open' flux could also be viewed as loops that 'close' in the outer boundary regions of the heliosphere, at over 100 AU from the Sun.

Open flux could be concentrated in a very few regions with quasi-steady conditions, as predicted by current-free models that describe the lowest energy state for a given photospheric boundary, or it could be distributed more evenly over the Sun with open fields and closed loops able to collide frequently and reconnect so that an open field closes while a closed field opens. If there are such frequent topology changes, then they could allow loop material to gain access to the heliosphere. The team will focus on the plausibility and consequences of this intrinsically time dependent evolution of the open flux distribution. Can it explain the distribution of ion abundances seen in the solar wind? Do the interactions of open and closed flux contribute to coronal heating?

The second question to be addressed is: How does the coronal magnetic flux change over time? The time dependence of the open flux distribution has to be accounted for in all models that connect the Sun and the heliosphere. Two major timescales are of interest: 40 hours, the time it takes the photosphere to replenish its magnetic field, and 11 years, the approximate time for each global reversal in magnetic polarities. Can the model based on frequent reconnections involving loops account for the magnetic polarity change of the Sun every 11 years? Or does the open flux at each pole flow across the equator as a continent-size patch toward the opposite pole, somewhat reminiscent of plate tectonics on Earth? Answering these questions will require more sophisticated computer modeling and a closer look at the behavior of coronal holes, which are the tracers of open fields in the corona.

The third question is: What are the physical processes governing these changes? Most models proposed to explain the evolving coronal field distribution invoke a combination of gradual but steady flows in the

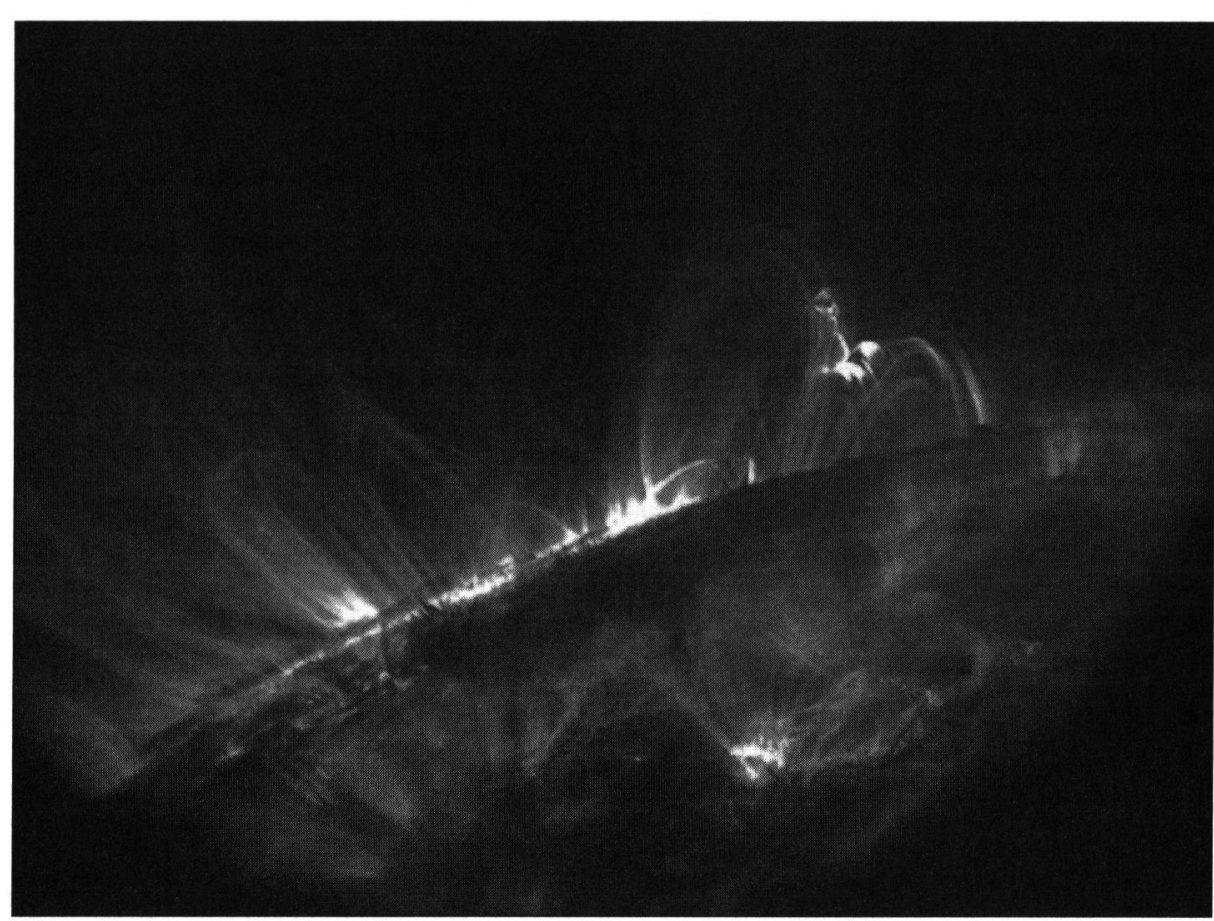

Fig. 1. A view of the solar corona where temperatures average 1,000,000K. Magnetic loops of various sizes and magnetic fields that open out to the heliosphere dominate the fine structure of the corona. Provided by the Transition Region and Coronal Explorer (TRACE) spacecraft (courtesy of TRACE team). Original color image appears at the back of this volume.

photosphere associated with the Sun's rotation, which varies with latitude, and meridional flows of about 10 meters per second from the equator to the poles. There are also random, convective flows in the photosphere that completely redistribute the fields every $\sim 40$ hours.

Additionally, the models must take into account the eruption of magnetic dipoles in many places on the Sun, especially the major eruptions that lead to the formation of sunspot groups. The effects of these photospheric events on the corona are not understood. Almost certainly, the transport processes depend on magnetic reconnection, as suggested by the coronagraph observations, which involves topological exchanges between open and closed fields and transfers of helicity from braided fields to twisted ones. But agreed-upon estimates as to where and at what speed these reconnection processes occur have not been formalized, and so it is difficult to gauge their contributions to coronal heating and acceleration. However, the topology changes may leave an imprint in the structure of sunspot regions and in the turbulence spectrum of the solar wind magnetic and velocity fields.

Finally, the team will ask: How can knowledge of coronal and photospheric processes lead to predictions of physical conditions in the heliosphere? The fundamental goal of the research is to develop an integrated approach that will lead to predictions of the structure of the heliospheric field and its development over time. This will include the integration of the answers to questions 1-3 into a coherent model whose predictions can be rigorously tested.
Figure 2 shows some initial results of one such approach. The model uses observed solar magnetic fields and calculates the overall topology of the heliospheric fields using a numerical solution of magnetohydrodynamic (MHD) equations. Figure 2 shows the results of two calculations, one for September 1996, near solar minimum, and one for November 2000, near solar maximum. The calculations make predictions for the location of the heliospheric current sheet, which separates outward pointing from inward pointing magnetic fields, which can be tested with interplanetary field measurements.

\section{Methodology for Addressing the Scientific Questions}

As explained in the introduction, the purpose of this focus group is to tackle these problems as a team. In order for this effort to be successful, the team approach is necessary. Also, a successful methodology has to involve models as a means to connect the different physical regions and communicate between the solar and heliospheric physics communities. Of course, it will require solar and heliospheric data as a reality check on the models. All these aspects are represented in the list of selected proposals (http://lws-trt. gsfc.nasa.gov/lws_abstract05.htm).

The proposed approaches include (1) models that follow the motion of the photospheric foot points of the open field and (2) models that examine the role of magnetic reconnection in coronal dynamics. Other approaches are (3) to determine turbulent energy dissipation in the corona in regimes with differing 


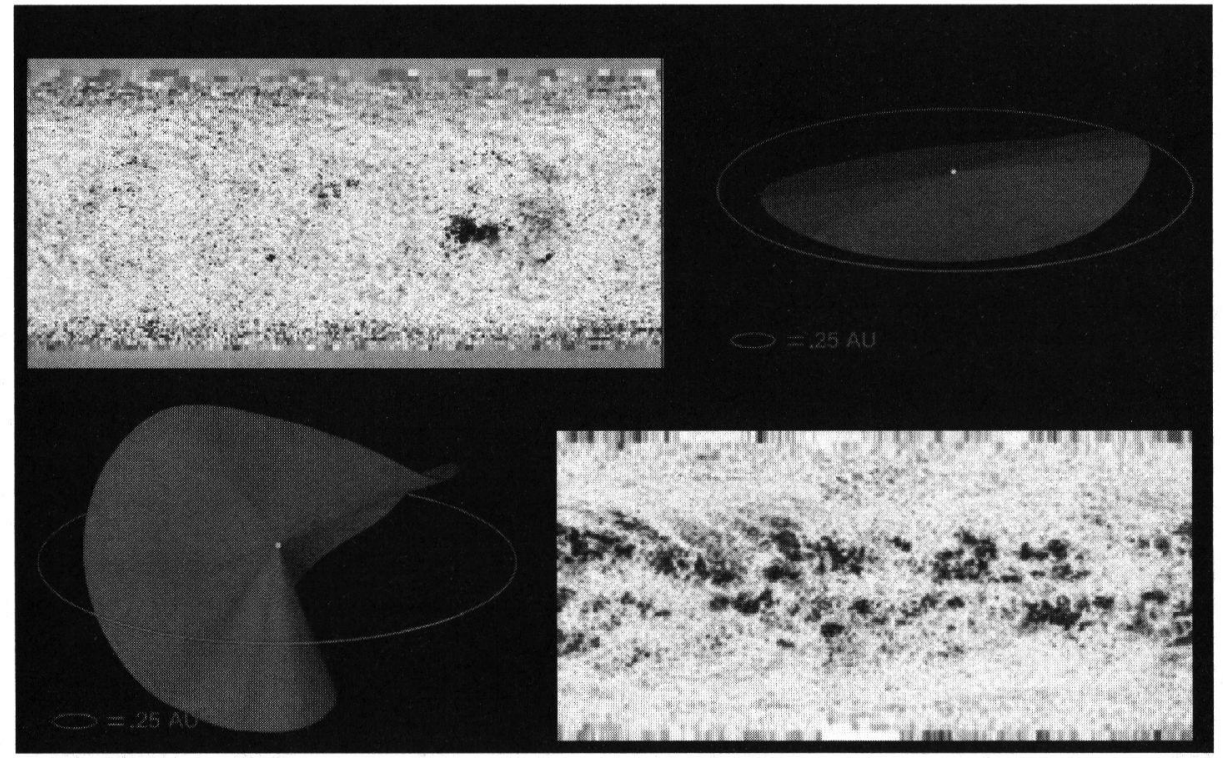

Fig. 2. Sun-heliosphere connections calculated with an MHD model. The model uses the observed distribution of solar photospheric magnetic fields, shown here in Mercator projections (top left and bottom right), at sunspot minimum and sunspot maximum, respectively. It calculates the extension of the fields into the heliosphere. The top right image shows the predicted structure of the heliospheric current sheet, which lies mostly in the ecliptic plane, denoted by the ellipse, at sunspot minimum. The image at bottom left shows the highly contorted current sheet typical of solar maximum conditions. MHD models have been successful at large scales. (Field data courtesy of Wilcox Solar Observatory, MHD model by SAIC Solar Group.) Original color image appears at the back of this volume.

magnetic field topology, (4) to determine if the structure of magnetic fields in the photosphere is correlated with the production of solar flares, (5) to model the decay of sunspot regions to improve understanding of the evolution of the patterns in the global fields, and (6) to see if the current-free model of coronal fields can be used to calculate the rate of opening up and closing down of open flux.

Identifying specific open fields in the heliosphere with their origins in the corona is important and challenging, so the team will (7) trace beams of electrons accelerated in solar eruptions back into the corona, where images may allow the electrons' injection point at the base of the open field to be pinpointed.

The Targeted Research and Technology focus team has decided to achieve coordination through a two-phase process that is enabled through a variety of electronic and traditional communications and regular meetings. Phase 1 is a 'collaborative phase' in which small interdisciplinary teams form and address issues related to questions 1-3 above and will last for approximately 1 year. Phase 2 is the 'integration phase' in which the focus team attempts to integrate knowledge to provide predictions of the changing heliosphere. The team meets twice a year in Boulder, Colo., and it uses teleconferences every 2 months or so to assess its progress and facilitate coordination between team members.

The team has established a Web library that includes a depository of presentations and discussions participated in by various members of the team through e-mails and chat rooms. Community members who are interested in following this work are encouraged to contact the team lead by sending an e-mail to thomasz@umich.edu

\section{Acknowledgments}

The Heliospheric Magnetic Field Focus Team consists of V. Abramenko (Big Bear Solar Observatory), S. Antiochos (Naval Research Laboratory), M. DeRosa (Lockheed Martin Solar and Astrophysics Laboratory), L.A. Fisk (University of Michigan), J. Gosling (Colorado University), D. Haggery (Johns Hopkins University/Applied Physics Laboratory), S. Lepri (U-M), J. Linker (Science Applications International Corporation), R. Lionello (SAIC), N. Murphy (Jet Propulsion Laboratory), M. Opher (George Mason University), D. Odstrcil (National Oceanic and Atmospheric Administration), D. Rust (JHU/ APL), K. Schrijver (LMSAL), N. Sheeley (NRL), B. Vasquez (University of New Hampshire), M. Velli (JPL), Y. Wang (NRL), V. Yurchyshyn (BBSO), and T.H. Zurbuchen (U-M).

\section{Author Information}

Thomas H.Zurbuchen, Lead of the Heliospheric Magnetic Field Targeted Research and Technology Focus Team, University of Michigan,Ann Arbor; E-mail: thomasz@umich.edu 


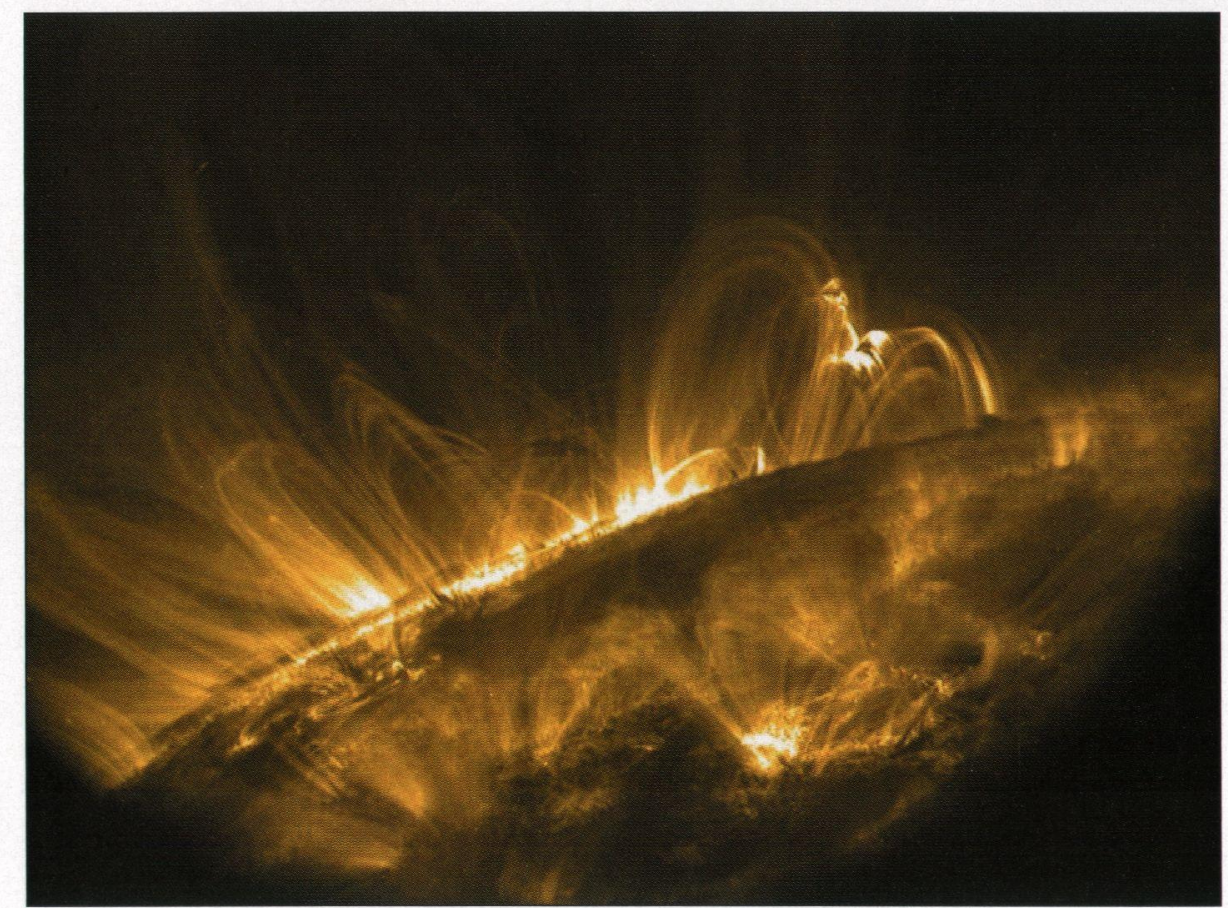

Fig. 1. A view of the solar corona where temperatures average 1,000,000K. Magnetic loops of various sizes and magnetic fields that open out to the heliosphere dominate the fine structure of the corona. Provided by the Transition Region and Coronal Explorer (TRACE) spacecraft (courtesy of TRACE team).

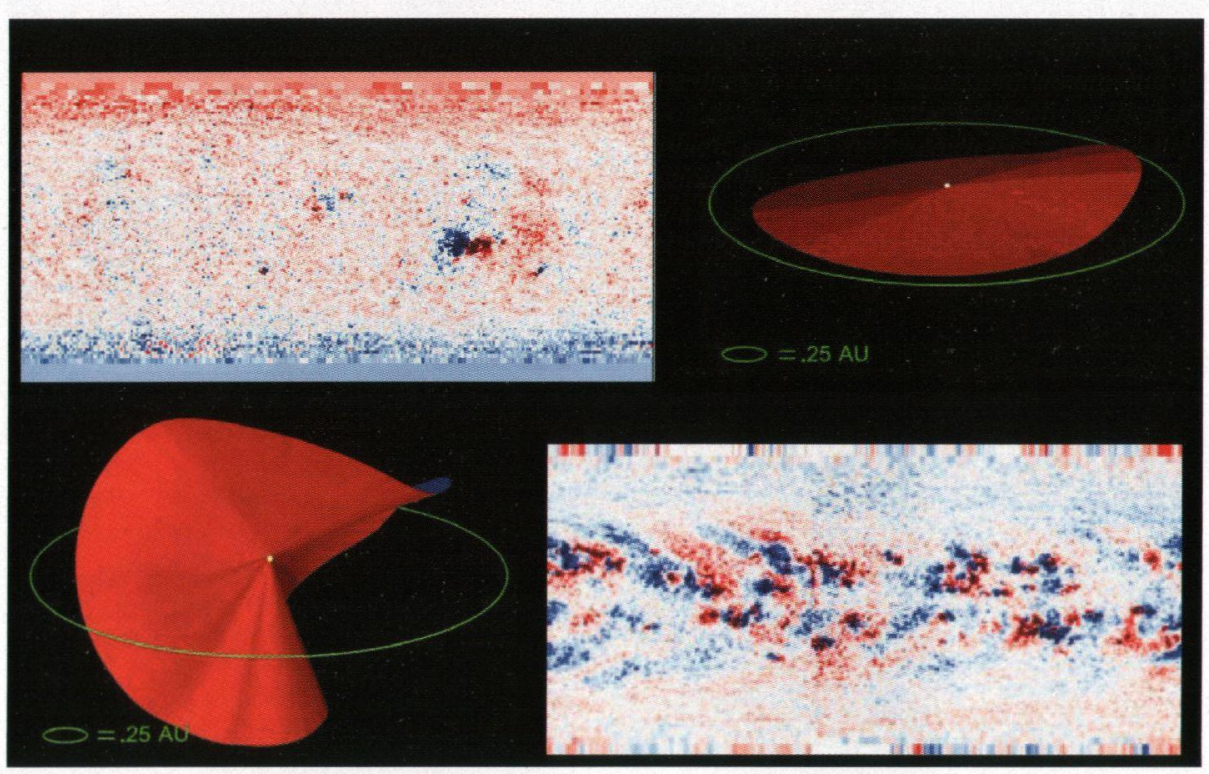

Fig. 2. Sun-heliosphere connections calculated with an MHD model.The model uses the observed distribution of solar photospheric magnetic fields, shown here in Mercator projections (top left and bottom right, with positive fields in red and negative ones in blue), at sunspot minimum and sunspot maximum, respectively. It calculates the extension of the fields into the heliosphere. The top right image shows the predicted structure of the heliospheric current sheet, which lies mostly in the ecliptic plane, denoted by the green ellipse, at sunspot minimum. The image at bottom left shows the highly contorted current sheet typical of solar maximum conditions. MHD models have been successful at large scales. (Field data courtesy of Wilcox Solar Observatory, MHD model by SAIC Solar Group.) 